\title{
Initial management of pneumonia and sepsis: factors associated with improved
} outcome

\author{
R. Menéndez*, A. Torres* ${ }^{\#}$, S. Reyes*, R. Zalacain`, A. Capelastegui+, J. Aspa ${ }^{\S}$, \\ L. Borderías ${ }^{\dagger}$, J.J. Martín-Villasclaras**, S. Bello ${ }^{\# \#}$, I. Alfageme ${ }^{\uparrow \uparrow}$, F.R. de Castro ${ }^{++}$, \\ J. Rello ${ }^{\S}$, L. Molinos ${ }^{f f}$ and J. Ruiz-Manzano***
}

ABSTRACT: Processes of care and adherence to guidelines have been associated with improved survival in community-acquired pneumonia (CAP). In sepsis, bundles of processes of care have also increased survival. We aimed to audit compliance with guideline-recommended processes of care and its impact on outcome in hospitalised CAP patients with sepsis.

We prospectively studied 4,137 patients hospitalised with CAP in 13 hospitals. The processes of care evaluated were adherence to antibiotic prescription guidelines, first dose within $6 \mathrm{~h}$ and oxygen assessment. Outcome measures were mortality and length of stay (LOS).

Oxygen assessment was measured in 3,745 (90.5\%) patients; 3,024 (73.1\%) patients received antibiotics according to guidelines and 3,053 (73.8\%) received antibiotics within $6 \mathrm{~h}$. In CAP patients with sepsis, the strongest independent factor for survival was antibiotic adherence (OR 0.4). In severe sepsis, only compliance to antibiotic adherence plus first dose within $6 \mathrm{~h}$ was associated with lower mortality (OR 0.60), adjusted for fine prognostic scale and hospital. Antibiotic adherence was related to shorter hospital stay.

In sepsis, antibiotic adherence is the strongest protective factor of care associated with survival and LOS. In severe sepsis, combined antibiotic adherence and first dose within $6 \mathrm{~h}$ may reduce mortality.

KEYWORDS: Audit, community-acquired pneumonia, compliance, length of stay, mortality, status

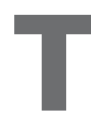
he incidence of community-acquired pneumonia (CAP) ranges from two to five cases per 1,000 persons $[1,2]$ and it is the main cause of death due to infection worldwide [2]. Severe sepsis is a major healthcare problem due to its high mortality, with the main cause being CAP in hospitalised patients. In fact, up to $70 \%$ of hospitalised CAP patients initially have sepsis or may develop sepsis during their hospital stay $[3,4]$.

The purpose of CAP guidelines and initiatives, such as the Surviving Sepsis Campaign (www. survivingsepsis.org), is to provide recommendations aimed at improving patient care. Indeed, implementation of these guidelines is associated with improved quality outcomes [5-8]. The recommendations of the most recent evidence-based guidelines include several processes of care linked to improved prognosis [9-11]. In the CAP guidelines, the three most recommended processes of care are adherence to antibiotic guidelines, first dose within $6 \mathrm{~h}$ and oxygen assessment reflecting care on admission. In an audit of quality of care in hospitals in the USA [12], blood cultures, smoking cessation advice and pneumococcal vaccination on discharge were also included. Nevertheless, in the case of blood cultures, current recommendations are aimed more at identifying a target population with a higher diagnostic yield than at a universal indication [13].

Several prior studies have demonstrated improvements in patient outcomes based on bundles of processes of care both in CAP $[5,6,14-16]$ and in sepsis [17, 18]. However, despite the interest in analysing the processes of care, their potential impact on prognosis is still a subject of debate [19, 20]. We hypothesised that compliance with guidelines on the processes of care is related to better outcomes, including mortality and length of stay (LOS) in CAP with sepsis or severe sepsis.
AFFILIATIONS

*Servicio de Neumología, Hospital Universitario La Fe, Ciberes, Valencia,

${ }^{\#}$ Servei de Pneumologia, Institut Clinic del Torax, IDIBAPS, Universitat de Barcelona, Ciberes GRS,

${ }^{\S \S}$ Critical Care, Hospital Vall d'Hebron, Institut de Recerca Vall d'Hebron-UAB, Ciberes, Barcelona, - Servicio de Neumología, Hospital de Cruces, Bilbao,

+Servicio de Neumología, Hospital de Galdakao, Galdakao,

${ }^{\S}$ Servicio de Neumología, Hospital de la Princesa, Madrid,

${ }^{f}$ Servicio de Neumología, Hospital San Jorge, Huesca,

**Servicio de Neumología, Hospital Carlos Haya, Malaga,

\#\# Servicio de Neumología, Hospital Miguel Servet, Zaragoza,

" Servicio de Neumología, Hospital de Valme, Sevilla,

++Servicio de Neumología, Hospital Dr. Negrín, Las Palmas de Gran Canaria,

${ }^{f f}$ Servicio de Neumología, Hospital Central Asturias, Oviedo, and

***Servicio de Neumología, Hospital Germans Trias i Pujol, Badalona,

Spain.

\section{CORRESPONDENCE}

R. Menéndez, Servicio de Neumología, Hospital Universitario i Politèctic Butevar sur s/n, 46026

Valencia, Spain

E-mail: rmenend@separ.es

Received:

Dec 072010

Accepted after revision:

July 042011

First published online:

Aug 042011

Online ISSN 1399-3003 
Our primary objective was to evaluate compliance with guidelines on processes of care and the effect of each or several combinations in patients hospitalised for CAP with sepsis and severe sepsis. A second goal was to investigate its impact on survival and LOS. We selected processes of care that depend on the care provider (physicians and nurses). Furthermore, we aimed to identify the most important combinations of processes of care that affect outcome measures.

\section{PATIENTS AND METHODS}

\section{Design and study population}

A prospective, multicentre, observational study was carried out from November 2005 to November 2007 in 13 hospitals belonging to the Spanish National Health System. Inclusion criteria were a new radiographic infiltrate compatible with the presence of acute pneumonia and at least two signs or symptoms of CAP. Exclusion criteria were admission within the previous 15 days, nursing-home patients, immunosuppressive treatment and/or steroids $\left(>15 \mathrm{mg} \cdot \mathrm{day}^{-1}\right)$ and do not resuscitate orders. The study was approved by the Ethics Committees (ISS, Hospital La Fe, Valencia, Spain; July 15, 2004) and the patients provided written informed consent.

We recorded data on age, sex, prior antibiotic treatment, adherence to guidelines, comorbidity and risk class according to FINE et al. [21]. Follow-up was performed after discharge to assess evolution and mortality after 30 days.

Sepsis and severe sepsis were defined according to previously accepted criteria $[3,22]$ Sepsis was defined as the presence of pneumonia and systemic inflammatory response syndrome $[3,22]$. Severe sepsis was considered if the criteria for sepsis were met, together with acute organ dysfunction: arterial hypoxaemia, creatinine $>2 \mathrm{mg} \cdot \mathrm{dL}^{-1}$, acute confusion, thrombocytopenia or hyperbilirubinaemia.

\section{Processes of care for in-patients}

The following processes of care in accordance with Spanish guidelines were recorded: 1) assessment of arterial oxygenation on presentation (by pulse oximetry or arterial blood gas analysis); 2) time until first antibiotic dose $(<6 \mathrm{~h})$; and 3$)$ antibiotic adherence to the Spanish guidelines [11]. Antibiotic adherence was considered as follows: in hospitalised CAP patients, either third-generation cephalosporin, amoxicillin-clavulanate combined with a macrolide, or third- or fourth-generation fluoroquinolone in monotherapy and, in intensive care unit patients, a combination of third-generation cephalosporin or amoxicillin-clavulanate plus macrolides or fluoroquinolone. All other regimens were considered nonadherent.

\section{Outcome measurements}

The outcome evaluated comprised mortality during hospitalisation and at 30 days.

LOS was defined as the number of days from admission to hospital to discharge.

\section{Statistical study}

Univariate analysis

Statistical analyses were performed using the SAS 8.2 software (SAS Institute, Inc., Cary, NC, USA). Categorical variables were compared using the Chi-squared test. Continuous variables were analysed using ANOVA or the Kruskal-Wallis test. Values of $p \leqslant 0.05$ were considered statistically significant. In order to evaluate the effect of several processes of care in combination, we stratified patients with one process of care (adherence to Spanish guidelines), two processes (antibiotic adherence to guidelines along with treatment within $6 \mathrm{~h}$ ) or three processes (antibiotic adherence, treatment within $6 \mathrm{~h}$ and oxygen assessment). LOS was dichotomised as short ( $\leqslant 7$ days) or long stay. Pneumonia severity index (PSI) was categorised as low risk (I-III risk classes) and high (IV-V) risk. Three Kaplan-Meier survival curves were constructed to assess the effect of processes of care and sepsis status on survival.

\section{Multivariate analysis}

Several logistic regression analyses were performed for each outcome: in-hospital and 30-day mortality, and LOS. For each dependent outcome variable, several logistic regression analyses were performed for the whole cohort and stratified by sepsis criteria using processes of care in one to three combinations as independent variables. We included the prognostic scale PSI and the hospital as independent variables in order to adjust for the independent effect of processes of care. The Hosmer and Lemeshow goodness-of-fit test was used to evaluate the adequacy of the models [23]. The areas under the receiver operating characteristic curves were also calculated.

\section{RESULTS}

We included 4,137 patients in our study after excluding 237 who lived in nursing homes: 2,966 (67.8\%) had sepsis and 1,572 $(38 \%)$ severe sepsis. The main demographic characteristics, comorbidities and PSI scores of the population are shown in table 1 .

The overall rates for the processes of care were as follows: 3,745 $(90.5 \%)$ patients had oxygen assessment; 3,024 (73.1\%) received antibiotics in accordance with the guidelines; and 3,053 (73.8\%) received the first dose of antibiotic within $6 \mathrm{~h}$ after arrival at the emergency department. The most frequent nonadherent regimens were: $\beta$-lactam monotherapy $(53 \%$ in the nonsepsis group, $46 \%$ in the sepsis group and $37 \%$ in the severe sepsis group) and fluorquinolone plus $\beta$-lactams $(27 \%$ in the nonsepsis group, $32 \%$ in the sepsis group and $36 \%$ in the severe sepsis group). The combination of two processes of care was observed in $53.4 \%$ of patients and three processes of care in $48.4 \%$ of patients (table 2 ).

Patients with severe sepsis were more likely to receive the first dose of antibiotic within $6 \mathrm{~h}$ and to have oxygen assessment, whereas compliance with antibiotic treatment recommended by the guidelines was significantly lower. Patients with severe sepsis had a higher probability of adherence to the three processes of care. Blood cultures were obtained in 645 (55.1\%) patients without sepsis, in 901 (64.6\%) of those with nonsevere sepsis and in $1,044(66.4 \%)$ of those with severe sepsis $(p<0.001)$.

\section{Outcome measures: univariate results}

A total of 167 (4\%) patients died during hospitalisation and 214 $(5.2 \%)$ died at 30 days. In-hospital mortality was $38(2.7 \%)$ in patients with nonsevere sepsis and $109(6.9 \%)$ in patients with severe sepsis, with the mortality at 30 days being 51 (3.8\%) and $131(8.5 \%)$, respectively. Adherence to the different isolated or combined processes of care with respect to mortality are shown in table 3. 
TABLE 1 Demographic characteristics, comorbidities and pneumonia severity index (PSI) scores

\begin{tabular}{|c|c|c|c|c|}
\hline & \multirow[t]{2}{*}{ Total } & \multicolumn{2}{|c|}{ Sepsis } & \multirow[t]{2}{*}{ p-value ${ }^{\#}$} \\
\hline & & Nonsevere sepsis & Severe sepsis & \\
\hline Subjects n & 4137 & $1394(33.7)$ & $1572(38.0)$ & \\
\hline Age yrs & $65.6 \pm 18.2$ & $61.5 \pm 19.3$ & $68.7 \pm 16.5$ & $<0.001$ \\
\hline Males/females & $2740(66.2) / 1397(33.8)$ & $909(65.2) / 485(34.8)$ & $1091(69.4) / 481(30.6)$ & 0.01 \\
\hline Influenza vaccination & 1749 (42.3) & $513(36.8)$ & $727(46.2)$ & $<0.001$ \\
\hline \multicolumn{5}{|l|}{ Comorbidity } \\
\hline Diabetes & $875(21.2)$ & $204(14.6)$ & $305(19.4)$ & 0.001 \\
\hline Liver disease & $176(4.3)$ & $49(3.5)$ & $73(4.6)$ & 0.1 \\
\hline Cardiopathy & $591(14.3)$ & $148(10.6)$ & $239(15.2)$ & $<0.001$ \\
\hline CNS disease & $422(10.2)$ & $126(9.0)$ & $170(10.8)$ & 0.1 \\
\hline
\end{tabular}

In-hospital mortality was significantly lower in patients with nonsevere sepsis who adhered to a process of care (antibiotic adherence) compared with patients who did not. An increase in the number of indicators did not reduce mortality. In patients with severe sepsis, the mortality was significantly lower (inhospital and 30-day mortality) in those with adherence to at least two indicators (table 3). LOS (expressed as median (interquartile range)) was analysed, excluding patients in the whole group who died and those in the groups with severe and nonsevere sepsis who died, according to adherence to the different combinations of processes of care (table 4).

LOS was found to be 1 day shorter in patients with nonsevere sepsis and adherence to one or more processes of care. Statistically significant differences were not found in the group with severe sepsis (tables 4 and 5).

In the group of patients with adherence to antibiotic treatment, we analysed the impact on mortality when the effect of other processes of care was added. The mortality tended to be lower when the first antibiotic dose was administered within the first $6 \mathrm{~h}(\mathrm{p}=0.053)$ (table 4). Furthermore, the Kaplan-Meier survival curves comparing survival of patients according to sepsis status, the effect of antibiotic adherence and timing within $6 \mathrm{~h}$ are provided in figure 1 . The survival was significantly higher in patients with antibiotic compliance and timing $<6 \mathrm{~h}$ in nonsevere (log rank Mantel-Cox 9.01; $\mathrm{p}=0.01$ ) and severe sepsis (log rank Mantel-Cox 9.39; $\mathrm{p}=0.009)$.

\section{Multivariate analyses}

Several multivariate logistic regression analyses were carried out with the dependent variables (in-hospital mortality, mortality at 30 days and LOS). The independent variables were processes of care, entered as compliance with one (antibiotic adherence to guidelines), two or three processes of care (table 6).

Adherence to antibiotic guidelines was the strongest protective factor for mortality in the whole population and in patients with nonsevere sepsis, and adding further processes of care did not improve survival. In the group with severe sepsis, the odds ratio for the protective effect of the combination of two or three processes of care (ORs 0.6 and 0.62 , respectively) was

TABLE 2 Processes of care and community-acquired pneumonia with and without sepsis

\begin{tabular}{|c|c|c|c|c|c|}
\hline & Total & No sepsis & Nonsevere sepsis & Severe sepsis & p-value ${ }^{\#}$ \\
\hline Abx & $3024(73.1)$ & $881(75.8)$ & $1043(75.0)$ & $1100(70.1)$ & 0.003 \\
\hline Oxygen assessment & $3745(90.5)$ & $1050(89.7)$ & 1164 (83.5) & $1531(97.4)$ & $<0.001$ \\
\hline Abx plus timing & $2208(53.4)$ & $599(53.8)$ & $753(56.6)$ & $856(56.1)$ & 0.7 \\
\hline Abx plus timing plus oxygen assessment & $2004(48.4)$ & 539 (48.0) & $634(47.3)$ & $831(54.4)$ & $<0.001$ \\
\hline
\end{tabular}

Data are presented as $n$ (\%), unless otherwise stated. Abx: antibiotic adherence to guidelines; Timing: first antibiotic dose $<6 \mathrm{~h}$. \#: p-values are the comparison between severe and nonsevere sepsis. 


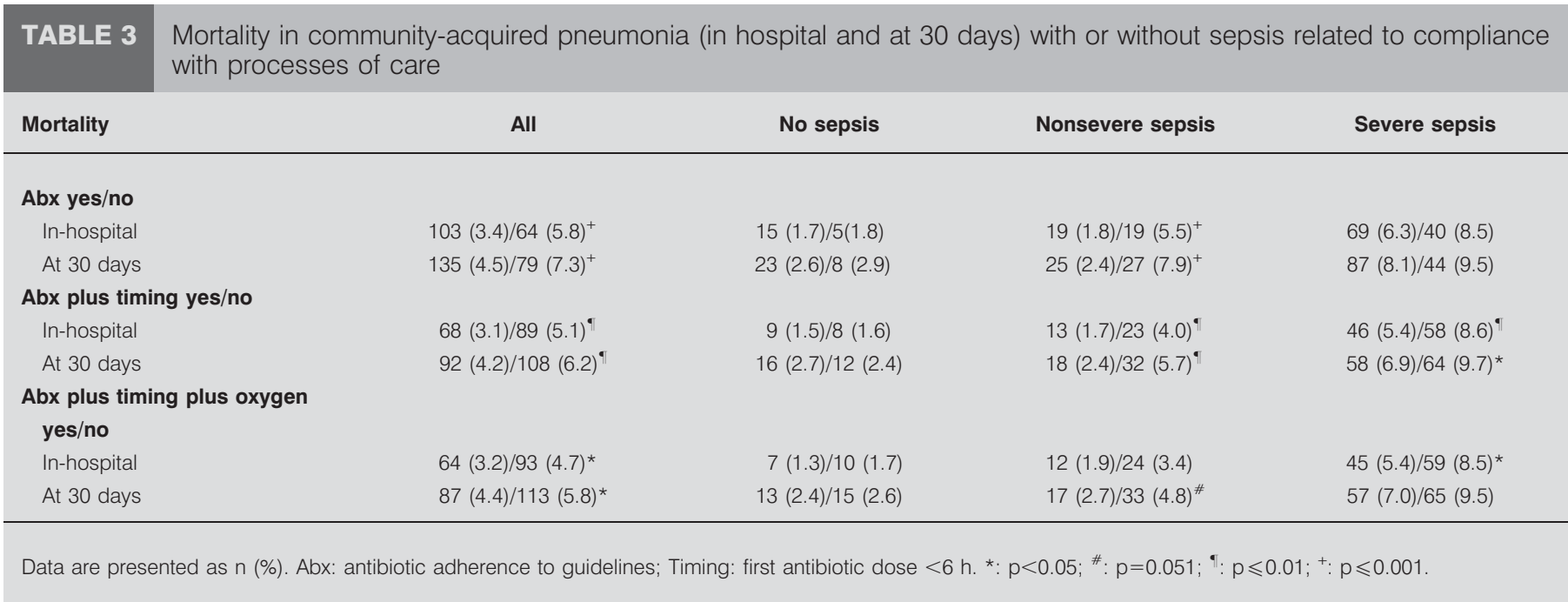

better than the odds ratio of a single process of care (OR 0.75) compared with in-hospital mortality. With regard to mortality at 30 days, only the odds ratio for two processes of care maintained a trend towards lower mortality $(p=0.06)$. The Chisquared goodness-of-fit analysis demonstrated the adequacy of the model $(\mathrm{p}=0.2)$.

In the multivariate models to predict a short stay ( $\leqslant 7$ days) in the whole population and in the group with nonsevere sepsis, a significant association was found with adherence to one, two or three processes of care (ORs in the sepsis group was $0.71,0.71$ and 0.61 , respectively). No significant association was found with any process of care in the subgroup with severe sepsis.

\section{DISCUSSION}

The most important findings of our study are as follows: 1) 38\% of the patients with hospitalised CAP had severe sepsis with a 30 -day mortality of $8.5 \%$ compared with $<2 \%$ in those without sepsis; 2 ) only $48 \%$ of patients were managed with adherence to three processes of care and oxygen assessment was not initially performed in $10 \%$; 3) in patients with nonsevere sepsis, adherence to antibiotic guidelines was the strongest independent protective factor associated with lower mortality and LOS; and 4 ) in patients with severe sepsis, the in-hospital mortality, adjusted for PSI and hospital effect, was only significantly lower when two processes of care (antibiotic adherence to guidelines and first dose within $6 \mathrm{~h}$ ) were followed.

In the present study, we have corroborated that severe sepsis increases mortality by CAP, although our figures of mortality are lower than those reported by DREMSIzOv et al. [3] (13.1\% versus $8.5 \%$ ), which is probably due to the exclusion of nursinghome patients, and are closer to those of SCHAAF et al. [4] in pneumococcal pneumonia. Both CAP and sepsis are frequent infectious causes of death worldwide and, thus, the interest in developing guidelines designed to clarify their management and make recommendations regarding all the steps to be taken is not surprising [11, 18, 24]. Processes of care that are dependent of the actions of healthcare professionals play a key role, as intervention directed to implement compliance is feasible. We found that the most commonly used process was oxygen assessment, and that adherence to antibiotic treatment and time until first dose guidelines were around $73 \%$. When the results were analysed for more than one process of care in the same patient, the percentage fell progressively, in line with the increase in processes. Thus, only $48 \%$ of patients received care that adhered to three processes, a figure similar to that found for sepsis by MIKKELSEN et al. [25]. Interestingly, this

TABLE 4 Impact of additional processes of care in patients with antibiotic adherence to Spanish guidelines

\begin{tabular}{|c|c|c|c|c|c|c|}
\hline \multicolumn{7}{|l|}{ Timing $<6 />6 \mathrm{~h}$} \\
\hline Severe sepsis & $46(5.4) / 18(9.0)$ & 0.053 & $58(6.9) / 20(10.2)$ & 0.1 & $8(5-13) / 7(5-11)$ & 0.2 \\
\hline \multicolumn{7}{|c|}{ Oxygen assessment yes/no } \\
\hline Nonsevere sepsis & $18(2.0) / 1(0.6)$ & 0.2 & $24(2.8) / 1(0.6)$ & 0.1 & $6(4-9) / 7(4-10)$ & 0.08 \\
\hline Nonsevere sepsis & $12(1.9) / 5(1.4)$ & 0.6 & $17(2.7) / 6(1.7)$ & 0.3 & $6(4-9) / 7(4-10)$ & 0.003 \\
\hline Severe sepsis & $45(5.4) / 19(8.4)$ & 0.09 & $57(7.0) / 21(9.5)$ & 0.2 & $8(5-13) / 7(5-12)$ & 0.3 \\
\hline
\end{tabular}

Data are presented as $n(\%)$ or median (interquartile range), unless otherwise stated. LOS: length of stay; Timing: first antibiotic dose <6 $\mathrm{h}$. 
TABLE 5 Length of stay in days and processes of care

\begin{tabular}{lllll} 
& \multicolumn{1}{c}{ All } & No sepsis & Nonsevere sepsis & Severe sepsis \\
\hline Abx yes/no & $7(4-10) / 7(5-11)^{*}$ & $6(4-9) / 7(5-10)^{* *}$ & $6(4-9) / 7(5-11)^{*}$ & $8(5-12) / 8(6-12)$ \\
Abx plus timing yes/no & $7(4-10) / 7(5-10)^{*}$ & $6(4-9) / 7(5-10)^{*}$ & $6(4-9) / 7(5-10)^{*}$ & $8(5-12) / 8(5-12)$ \\
Abx plus timing plus oxygen yes/no & $7(4-10) / 7(5-10)^{*}$ & $6(4-9) / 7(5-9)^{*}$ & $6(4-8) / 7(4-10)^{*}$ & $8(5-12) / 8(5-12)$ \\
\hline
\end{tabular}

Data are presented as median (interquartile range). Abx: antibiotic adherence to guidelines; Timing: first antibiotic dose $<6 \mathrm{~h} .{ }^{*}: \mathrm{p}=0.057 .{ }^{*}: \mathrm{p}<0.05 ;{ }^{* *}: \mathrm{p}<0.01$.

percentage was higher in patients with severe sepsis $(54.4 \%)$, with more patients treated within the first $6 \mathrm{~h}$, although with lower antibiotic adherence (mainly due to a broader antibiotic spectrum).

Our findings show that antibiotic adherence to Spanish guidelines and first dose within $6 \mathrm{~h}$ were associated with a lower mortality (in-hospital and at 30 days) and a lower LOS in the whole cohort. Interestingly, antibiotic adherence was the strongest protective factor for in-hospital mortality and mortality at 30 days in patients with nonsevere sepsis, and a further process of care did not improve outcome. Conversely, in CAP with severe sepsis, the in-hospital mortality was only significantly reduced when both processes of care were applied.
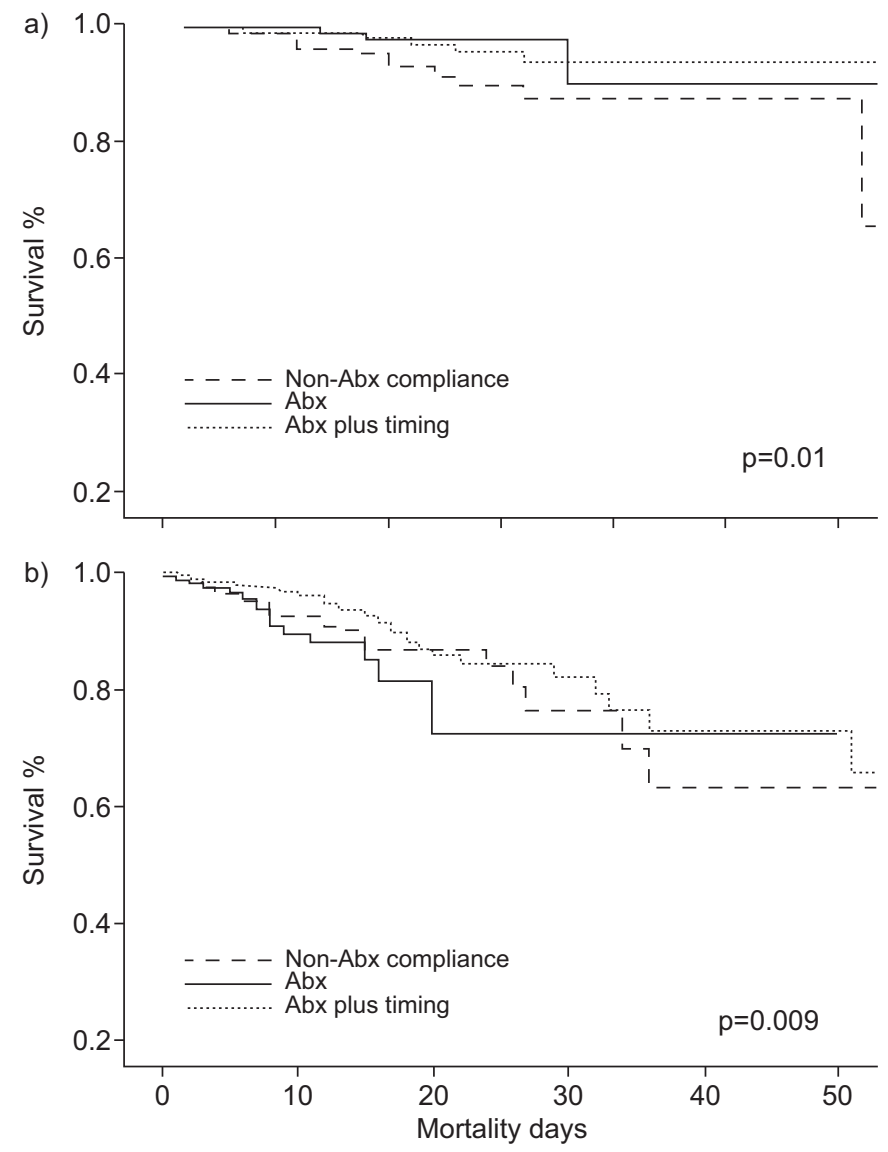

FIGURE 1. Kaplan-Meier survival curves in a) nonsevere and b) severe sepsis community-acquired pneumonia patients comparing the effect of antibiotic compliance and timing $<6 \mathrm{~h}$. Abx: antibiotic adherence to guidelines.
Adherence to antibiotic treatment is the process of care that has most consistently shown a positive effect in many studies $[6,7,26-28]$. Moreover, in the subset of patients who received guideline-adherent antibiotics, treatment within the first $6 \mathrm{~h}$ was found to significantly reduce mortality. Although it has been shown that a shorter time to antibiotic initiation improves outcome [19], some authors have pointed out the harmful consequences of this measure, such as inappropriate use of antibiotics before confirming diagnosis [29, 30]. Despite the debate regarding the number of hours, we corroborated the fact that when antibiotic is administered within $6 \mathrm{~h}$, several outcome measurements are improved (in-hospital mortality in severe sepsis $(\mathrm{p}=0.05)$ and lower LOS in sepsis $(\mathrm{p}<0.05))$. This effect was specifically shown in patients with severe sepsis who received guideline-adherent antibiotics, which is precisely where the effect should be most evident.

Curiously, oxygen assessment has been found to be associated with higher mortality and poorer prognosis. The apparent discrepancy between this process of care and prognosis can be explained by the fact that the lack of this assessment corresponds to younger patients without comorbid conditions and, consequently, a lower mortality. In fact, in patients with severe sepsis, oxygen assessment performed with in nearly $98 \%$ of cases and it has been reported that early assessment in severe pneumonia improves survival [31].

The best combination of processes of care in hospitalised patients with CAP and severe sepsis, as confirmed by the multivariate analysis, includes at least two processes of care (antibiotic adherence and treatment within the first $6 \mathrm{~h}$ ). However, in less severe patients with nonsevere sepsis and a single process of care (antibiotic adherence), the protective factor is very similar to that of several processes of care. This finding confirms the importance of the impact of quality and the effect of two concomitant processes of care in patients with severe sepsis. Bundles of care have been shown to increase survival in patients with sepsis of different aetiologies [17, 32]. Our findings, at least in the subset of patients with severe sepsis, contradict the recent recommendations of the American Academy of Emergencies that consider it useless to measure the time until the first dosage of antibiotics in CAP [30].

LOS is an end-point that depends on several factors related to the patient, comorbidities and social factors [33,34]. However, in the whole cohort and in patients with sepsis, we consistently found that the median LOS was 1 day shorter if antibiotic use adhered to guidelines $[35,36]$.

One limitation of our study is that the observational design makes it difficult to establish a cause-effect relationship between 


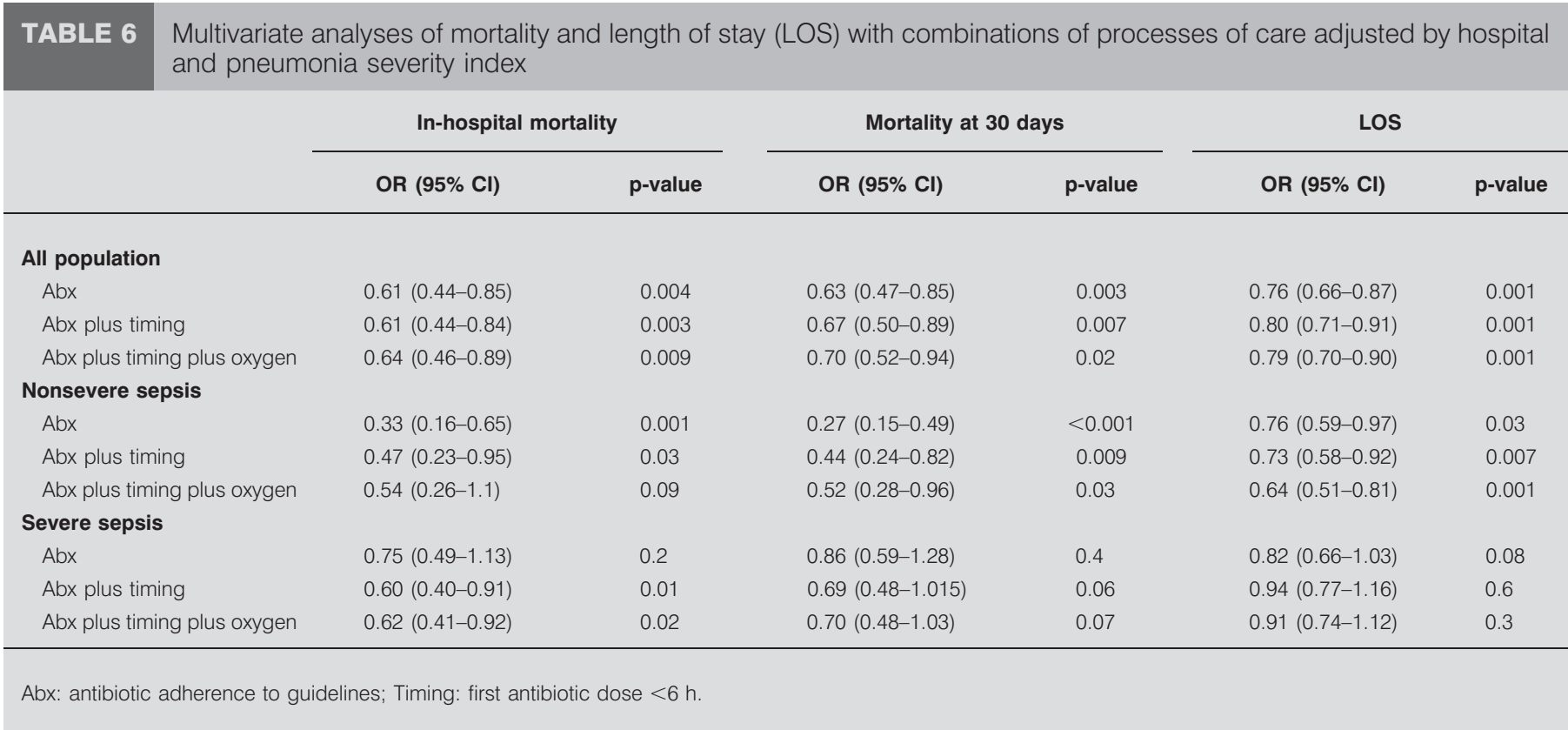

processes of care and prognosis. Their effect on outcome could be due to changes in unmeasured processes of care or better medical care [37]. While it is difficult to attribute improvements in outcome to improvements in quality of care, some investigators have demonstrated improvements based on implementation of bundles of processes of care [37]. Additional measures, such as the efficiency of care during hospitalisation, are clearly needed, despite being difficult to describe.

In summary, we have confirmed that there are areas where improvement is needed to increase quality care in hospitalised patients with CAP, mainly in those with severe sepsis. In CAP with severe sepsis, selection of an antibiotic and timing of the first dose within $6 \mathrm{~h}$ may reduce mortality, whereas in nonsevere sepsis, antibiotic selection is more decisive. Our findings might have clinical implications in managing hospitalised CAP in the emergency room. Efforts should be directed toward identifying factors that affect poorer compliance with quality indicators in order to prepare specific strategies for their resolution.

\section{SUPPORT STATEMENT}

The study was supported by CIBERES, an initiative of ISCIII, FIS grant PI041150, SEPAR grant 2007 and PII (SEPAR Research Programme) in respiratory infections, and a grant from the Ministry of Health of the Autonomous Community of Valencia Consellería Sanitat Comunidad Valenciana 2007.

\section{STATEMENT OF INTEREST}

None declared.

\section{ACKNOWLEDGEMENTS}

The authors thank I. Arribas (ERATEMA Associate Director, Valencia, Spain) for his expert statistical contribution.

\section{REFERENCES}

1 Almirall J, Bolibar I, Vidal J, et al. Epidemiology of communityacquired pneumonia in adults: a population-based study. Eur Respir J 2000; 15: 757-763.
2 Kaplan V, Angus DC, Griffin MF, et al. Hospitalised communityacquired pneumonia in the elderly: age- and sex-related patterns of care and outcome in the United States. Am J Respir Crit Care Med 2002; 165: 766-772.

3 Dremsizov T, Clermont G, Kellum JA, et al. Severe sepsis in community-acquired pneumonia: when does it happen, and do systemic inflammatory response syndrome criteria help predict course? Chest 2006; 129: 968-978.

4 Schaaf B, Kruse J, Rupp J, et al. Sepsis severity predicts outcome in community-acquired pneumococcal pneumonia. Eur Respir J 2007; 30: 517-524.

5 Capelastegui A, Espana PP, Quintana JM, et al. Improvement of process-of-care and outcomes after implementing a guideline for the management of community-acquired pneumonia: a controlled before-and-after design study. Clin Infect Dis 2004; 39: 955-963.

6 Dean NC, Bateman KA, Donnelly SM, et al. Improved clinical outcomes with utilization of a community-acquired pneumonia guideline. Chest 2006; 130: 794-799.

7 Menendez R, Torres A, Zalacain R, et al. Guidelines for the Treatment of Community-acquired Pneumonia: Predictors of Adherence and Outcome. Am J Respir Crit Care Med 2005; 172: 757-762.

8 Arnold FW, LaJoie AS, Brock GN, et al. Improving outcomes in elderly patients with community-acquired pneumonia by adhering to national guidelines: Community-Acquired Pneumonia Organization International cohort study results. Arch Intern Med 2009; 169: 1515-1524.

9 Niederman MS, Mandell LA, Anzueto A, et al. Guidelines for the management of adults with community-acquired pneumonia. Diagnosis, assessment of severity, antimicrobial therapy, and prevention. Am J Respir Crit Care Med 2001; 163: 1730-1754.

10 Bartlett JG, Dowell SF, Mandell LA, et al. Practice guidelines for the management of community-acquired pneumonia in adults. Infectious Diseases Society of America. Clin Infect Dis 2000; 31: 347-382.

11 Alfageme I, Aspa J, Bello S, et al. [Guidelines for the diagnosis and management of community-acquired pneumonia. Spanish Society of Pulmonology and Thoracic Surgery (SEPAR)]. Arch Bronconeumol 2005; 41: 272-289.

12 Williams SC, Schmaltz SP, Morton DJ, et al. Quality of care in U.S. hospitals as reflected by standardized measures, 2002-2004. N Engl J Med 2005; 353: 255-264. 
13 Falguera M, Trujillano J, Caro S, et al. A prediction rule for estimating the risk of bacteremia in patients with communityacquired pneumonia. Clin Infect Dis 2009; 49: 409-416.

14 Marrie TJ, Lau CY, Wheeler SL, et al. A controlled trial of a critical pathway for treatment of community-acquired pneumonia. CAPITAL Study Investigators. Community-Acquired Pneumonia Intervention Trial Assessing Levofloxacin. JAMA 2000; 283: 749-755.

15 McCabe C, Kirchner C, Zhang H, et al. Guideline-concordant therapy and reduced mortality and length of stay in adults with community-acquired pneumonia: playing by the rules. Arch Intern Med 2009; 169: 1525-1531.

16 Meehan TP, Weingarten SR, Holmboe ES, et al. A statewide initiative to improve the care of hospitalised pneumonia patients: the Connecticut Pneumonia Pathway Project. Am J Med 2001; 111: 203-210.

17 Ferrer R, Artigas A, Suarez D, et al. Effectiveness of treatments for severe sepsis: a prospective, multicenter, observational study. Am J Respir Crit Care Med 2009; 180: 861-866.

18 Dellinger RP, Carlet JM, Masur H, et al. Surviving Sepsis Campaign guidelines for management of severe sepsis and septic shock. Crit Care Med 2004; 32: 858-873.

19 Houck PM, Bratzler DW, Nsa W, et al. Timing of antibiotic administration and outcomes for medicare patients hospitalised with community-acquired pneumonia. Arch Intern Med 2004; 164: 637-644.

20 Waterer GW, Kessler LA, Wunderink RG. Delayed administration of antibiotics and atypical presentation in community-acquired pneumonia. Chest 2006; 130: 11-15.

21 Fine MJ, Auble TE, Yealy DM, et al. A prediction rule to identify low-risk patients with community-acquired pneumonia. $N$ Engl J Med 1997; 336: 243-250.

22 Levy MM, Fink MP, Marshall JC, et al. 2001 SCCM/ESICM/ ACCP/ATS/SIS International Sepsis Definitions Conference. Intensive Care Med 2003; 29: 530-538.

23 Hosmer D, Lemeshow S. Applied Logistic Regression. New York, John Wiley \& Sons, Inc., 1989.

24 American Thoracic Society, Infectious Diseases Society of America. Guidelines for the management of adults with hopsitalacquired, ventilator-associated and healthcare associated pneumonia. Am J Respir Crit Care Med 2005; 171: 388-416.

25 Mikkelsen ME, Gaieski DF, Goyal M, et al. Factors associated with nonadherence to early goal-directed therapy in the ED. Chest 2010; 138: 551-558.
26 Dambrava PG, Torres A, Valles X, et al. Adherence to guideline's empiric antibiotic recommendations and CAP outcome. Eur Respir J 2008; 32: 892-901.

27 Frei CR, Restrepo MI, Mortensen EM, et al. Impact of guidelineconcordant empiric antibiotic therapy in community-acquired pneumonia. Am J Med 2006; 119: 865-871.

28 Shorr AF, Bodi M, Rodriguez A, et al. Impact of antibiotic guideline compliance on duration of mechanical ventilation in critically ill patients with community-acquired pneumonia. Chest 2006; 130: 93-100.

29 Metersky ML, Sweeney TA, Getzow MB, et al. Antibiotic timing and diagnostic uncertainty in Medicare patients with pneumonia: is it reasonable to expect all patients to receive antibiotics within 4 hours? Chest 2006; 130: 16-21.

30 Pines JM, Isserman JA, Hinfey PB. The measurement of time to first antibiotic dose for pneumonia in the emergency department: a white paper and position statement prepared for the American Academy of Emergency Medicine. J Emerg Med 2009; 37: 335-340.

31 Blot SI, Rodriguez A, Sole-Violan J, et al. Effects of delayed oxygenation assessment on time to antibiotic delivery and mortality in patients with severe community-acquired pneumonia. Crit Care Med 2007; 35: 2509-2514.

32 Kumar A, Ellis P, Arabi Y, et al. Initiation of inappropriate antimicrobial therapy results in a fivefold reduction of survival in human septic shock. Chest 2009; 136: 1237-1248.

33 McCormick D, Fine MJ, Coley CM, et al. Variation in length of hospital stay in patients with community-acquired pneumonia: are shorter stays associated with worse medical outcomes? Am J Med 1999; 107: 5-12.

34 Menendez R, Cremades MJ, Martinez-Moragon E, et al. Duration of length of stay in pneumonia: influence of clinical factors and hospital type. Eur Respir J 2003; 22: 643-648.

35 Battleman DS, Callahan M, Thaler HT. Rapid antibiotic delivery and appropriate antibiotic selection reduce length of hospital stay of patients with community-acquired pneumonia: link between quality of care and resource utilization. Arch Intern Med 2002; 162: 682-688.

36 Capelastegui A, Espana PP, Quintana JM, et al. Declining length of hospital stay for pneumonia and postdischarge outcomes. Am J Med 2008; 121: 845-852.

37 Bratzler DW, Nsa W, Houck PM. Performance measures for pneumonia: are they valuable, and are process measures adequate? Curr Opin Infect Dis 2007; 20: 182-189. 